\title{
DE-FGO2-88ER60621
}

DOE/ER/60621--.5

DE92 013002

\section{REPAIR OF UV DAMAGED DNA: GENES AND PROTEINS OF YEAST AND HUMAN}

(New title)

\section{Progress Report}

for period November 1, 1991 - April 15, 1992

Louise Prakash

University of Rochester School of Medicine

Rochester, NY 14642

April 1992

Prepared for

THE U.S. DEPARTMENT OF ENER:GY

AGREEMENT NO. DE-FGO2-88ER60621

\section{NOTICE}

This report was prepared as an account of work sponsored by the United States Government. Neither the United States nor the Department of Energy, nor any of their employees, nor any of their contractors, subcontractors, or their employees, makes any warranty, express or implied, or assumes any legal liability or responsibility for the accuracy, completeness, or usefulness of any information, apparatus, product or process disclosed or represents that its use would not infringe privately-owned rights. 


\title{
PLEASE NOTE
}

This Progress Report, DOE/ER/60621-5 is for the grant entitled "Repair of UV damaged DNA: genes and proteins of yeast and human". Progress Report DOE/ER/6-621-4 was submitted last April, 1991. The previous title of this funded grant was "Excision repair of UV damaged DNA: genes and proteins of yeast and human." As instructed last year by my DOE Technical Monitor, George Duda, my approved pre-proposal "Structure and function of the human homologs of the yeast RAD6 and RAD 18 DNA repair genes: was treated as a uupplement to project DE-FGO288ER60621, and was given the new title indicated above ("Repair of UV damaged DNA: genes and proteins of yeast and human"). This title was used last year and will continue to be used in the future.

\begin{abstract}
Our objectives are to determine the molecular mechanism of the incision step of excision repair of ultraviolet (UV) lighi damaged DNA in eukaryotic organisms, using the yeast Saccharomyces cerevisiae as a model system, and to study the human homologs of yeast excision repair and postreplication repair proteins. We found that the phenotype of a deletion $(\Delta)$ mutation of $r h p 3^{+}$, the Schizosaccharomyces pombe homolog of RAD3 of Saccharomyces cerevisiae, is inviable, like the $S$. cerevisiae rad $3 \Delta$ mutation. S. pombe rhp $3^{+}$complements the inviability and UV sensitivity of $S$. cerevisiae rad $3 \Delta$ and rad3 point mutants, respectively, and $S$. cerevisiae $R A D 3$ complements both defects of the $S$. pombe rhp 3 mutants. Using a nitrocellulose filter assay, we have shown that RAD10 protein is a DNA-binding protein with strong preference for singlestranded DNA and that it promotes renaturation of complementary DNA strands. We found that the yeast $R A D 14$ gene, which functions in excision repair, is homologous to the human XPAC gene. Another human excision repair gene, $E R C C 3 / X P B C$, whose yeast homolog we designated $S C E R C C 3$, is essential for cell viability. Since the yeast and human ERCC3 encoded proteins are so homologous, we expect the human ERCC3/XPBC gene to be essential as well. Since defective DNA repair and enhanced neoplasia characterize several human genetic diseases, and repair proteins are highly conserved between yeast and man, a thorough understanding of the molecular mechanisms of DNA repair in yeast should provide a better understanding of the causes of cancer.
\end{abstract}

Time or effect devoted to project: The principal investigator has devoted $50 \%$ of effort on this project since the beginning of the current term of agreement and will d:yote $60 \%$ of her effort on the project during the remainder of the current term, to reflect the addition of the supplement, as discussed above in the note.

Goals of the project: This project originally dealt with the characterization of three $R A D$ genes and their encoded proteins involved in the incision step of excision repair followirig exposure to UV light in the eukaryotic organism, the yeast Saccharomyces cerevisiae. Since the beginning of the project, the goals have been expanded to include the response to preproposal project (approved last year) entitled "Structure and function of the human homologs of the yeast $R A D 6$ and $R A D 18$ DNA repair genes". The combined aims for the entire project period of November 1, 1990 to October 31, 1993 now are: (1) Studies of the $R A D B$ gene and protein - (a) to determine the nature of the defect in rad3 temperature sensitive mutants, (b) to further biochemically characterize the RAD3 protein (2) Studies of ERCC2, the human homolog of RAD3 - (a) to purify and biochemically characterize the human ERCC2 protein, (b) to determine the abilit, of the human $E R C C$ ? gene to functionally substitute for the excision repair and viability functions of the yeast $R A D 3$ gene (3) Studies with the RADIO gene and protein - (a) to isolate and characterize mutations of $R A D 10$ which differentially inactivate excision repair and mitotic recombination, (b) to determine the enzymatic activities associated with RAD10 protein (4) Studies with RAD1 protein - to purify and biochemically characterize RAD1 protein, as well as rad1 mutant proteins obtained from mutants which inactivate excision 
repair and mitotic recombination differentially (5) Studies with the yeast lomolog of the human $E R C C 3 / X P-B$ gene - to genetically characterize the yeast homolog of the human $E R C C 3 / X P-B$ gene, $S C E R C C 3$ and to purif $y$ and biochemically characterize its encoded protein (6) Combinations of RAD1, RAD3, ScERCC3 and RAD10 proteins - to determine whether the RAD1, RAD3, ScERCC3, and RAD10 proieins form a complex that functions in excision repair (7) The human homolog of the RAD18 genes of yeast - (a) to clone the human homolog of $R A D 18$, determine its nucleotide sequence, and examine whether it can functionally substitute for the S. cerevisiae $R A D 18$ gene, (b) to purify and characterize the human RAD18 protein, (c) since the $S$. cerevisiae RAD6 and RAD18 proteins co-immunoprecipitate, to determine whether the human RAD6 protein and human RAD18 proteins can substitute for their yeast homologs in the immune complex.

Description of the scope of investigations undertaken and the significant results obtained:

(1) rhp3+, the Schizosaccharomyces pombe homolog of the
Saccharomyces cerevisiae RAD3 gene

In addition to its requirement for excision repair, the $S$. cerevisiae $R A D 3$ gene is essential for cell viability. We showed previously that RAD3 protein possesses a single-stra.1ded DNAdependent ATPase and DNA and DNA.RNA helicase activities. Our mutational studies have indicated a requirement for the RAD3 helicase activities in excision repair. The RAD: and human $E R C C 2$ encoded proteins share $53 \%$ identity and $75 \%$ similarity. ERCC2 has re cently been shown to be the same as the human XPDC gene. In order to examine the extent of conservation of the structure and function of RAD3 during eukaryotis evolution, we cloned the RAD 3 homolog, rhp $3^{+}$, from the distantly related yeast Schizosaccharomyces pombe. RAD3 and rhp $3^{+}$encoded proteins show $67 \%$ identity and $82 \%$ similarity, when conservative amino acid substitutions are taken into account. The RAD3, rhp $3^{+}$, and ERCC2 proteins share extensive amino acid similarity throughout their length, but the homology is particularly strong in the seven conserved helicase domains.

Our genetic studies with the $r h p 3^{+}$gene establish that like $R A D 3$ of $S$. cerevisiae, rhp $3^{+}$is required for DNA repair and cell viability in $S$. pombe. The $r h p 3^{+}$gene complements the UV sensitivity of the $S$. pombe rad15 mutation, implying that rad15 is involved in excision repair. We mutated the lysine 48 residue to arginine in the conserved nucleotide binding sequence (GKT) of the rhp $3^{+}$protein in order to examine the role of the putative DNA helicase activity. The rhp3 Arg48 mutation inactivated the DNA repair function of $r h p 3^{+}$but had no effect on cell viability, indicating a requirement of $\mathrm{rhp} 3^{+}$helicase activities in DNA repair. These results are analogous to those we obtained with the corresponding rad3 Arg-48 mutant of $S$. cerevisiae.

Perhaps the most remarkable finding in this work is the extent to which RAD3 and $r h p 3^{+}$ genes functionally substitute for each other. The $S$. cerevisiae RAD3 gene fully complements the viability defect of the $S$. pombe rhp $3 \Delta$ mutant, restoring its growth to the wild type rate. $R A D 3$ also restores wild type levels of $\mathrm{JV}$ resistance to the $S$. pombe rad15 and rhp3 4 mutants. The reciprocal experiments show that $r h p 3^{+}$complements the DNA repair and viability defects of various rad3 mutants to wild type levels. 


\section{(2) The RAD10, protein}

We have further characterized the biochemical properties of RAD10 protein purified to near homogeneity from yeast cells carrying a plasmid containing an $A D C 1:: R A D 10$ fusion. Previously, we had obtained evidence for RAD10 binding to DNA by a gel retardation assay. Now, we have characterized the DNA-binding properties of RAD10 using a nitrocellulose filter binding assay. RAD10 protein shows a strong preference for binding to single-stranded DNA (ssDN/2) over double-stranded DNA (dsDNA). The percentage of ssDNA retained on alkali-treated filters is proportional to the amount of input of RAD10, reaching a maximum at a protein:DNA molar ratio of about one RAD 10 monomer per 50 nucleotides. Under the conditions used for optimal binding of RAD10 to ssDNA, RAD10 shows little binding to dsDNA. UV irradiation of DNA to $100 \mathrm{C}$ $\mathrm{Jm}^{-2}$ does not stimulate binding of RAD10 to either ss- or dsDNA. The middle portion of RAD10 contains many tyrosine residues and and is basic, and this region could be involved in binding to ssDNA by intercalation of tyrosine resiciues between the bases in DNA and through ionic interactions.

Since $\mathrm{FAD} 10$ has a role in genetic recombination, we determined whether RAD10 promotes the renaturation of homologous single strands of DNA using a gel assay. Incubation of heat-denature dsDNA with RAD10 results in a species of DNA that does not migrate into a $0.8 \%$ agarose gel. 'The high $\mathrm{M}_{\mathrm{r}}$ DNA product was not destabilized by stripping RAD10 from the DNA prior to electrophoresis by the addition of $1 \%$ SDS; however, boiling dissociates the DNA product into a form having the same mobility as the heai-denatured DNA. Substitution of noncomplementary M13 viral ssDNA does not result in formation of high $\mathrm{M}_{\mathrm{r}}$ DNA. If the dsDNA is not heat-denatured, formation of the high $\mathrm{M}_{\mathrm{r}}$ DNA product did not occur either. We also used an $\mathrm{S} 1$ nuclease digestion assay to examine RAD10-mediated renaturation of DNA and observe a RAD10 concentration-dependent conversion of heat-denatured T7 DNA into an S1 resistant form which reaches a near maximal level at a protein to DNA ration of one RAD10 monomer per 60 nucleotides.

\section{(3) RAD14 gene and protein}

Cell fusion studies have defined seven xeroderma pigmentosum complementation groups, $\mathrm{XP}-\mathrm{A}$ to XP-G. In S. cerevisiae, the genetic complexity of excision repair is the same as it is in humans. At least ten genes are required for excision repair in yeast. Mutations in any one of five yeast genes, $R A D 1, R A D 2, R A D 3, R A D 4$, and $R A D 10$, cause a total defect in incision and result in extreme sensitivity to $U V$. Of the remaining $R A D$ genes required for excision repair, the available rad14 point mutant is only moderately UV sensitive and carries out a substantial amount of incision of UV damaged DNA. Recently, we cloned and sequenced the RADI4 gene of $S$. cerevisiae.

The $R A D 14$ open reading frame encodes a highly hydrophilic protein of 247 amino acids with a predicted relative molecular mas of 29,328. RAD14 contains a zinc-finger motif from amino acid residues 67 to 92 and another potential metal-binding sequence between residues 207 and 238. Interestingly, RAD14 shows homology to the human XPAC protein which contains 273 amino acids. The homology is most extensive in the middle portion of the two proteins, between residues 67 to 198 of RAD14 and 105 to 235 of XPAC 'The two proteins share 33\% identity and $61 \%$ similarity in this region if identical and conserved esidues are grouped together. The 4cysteine zinc-finger motif in present in both proteins at the beginning of this homologous segment and the zinc-finger sequence in RAD14 and XPAC might be involved in DNA binding, or it might affect protein-protein interactions. Unlike the previously studied radl4-2 point mutation, the rad14 $\Delta$ mutation results in UV sensitivity equivalent to that of the totally incision-defective radl $\Delta$ mutant. To investigate further whether RAD14 is essential for the incision step of excision repair, we coupled the rad14 $\Delta$ mutation to the temperature-sensitive DNA ligase mutant $c d c 9-2$, and determined the incidence of incision breaks following UV irradiation. Our results indicate that $R A D 14$ is required for incision of UV damaged DNA, bringing the total of genes involved in this 
process in yeast to at least six. Double mutant analysis of rad14 $\Delta$ in combination with radl $\Delta$. rad6 $\Delta$ defective in postreplication repair, or $\operatorname{rad} 52 \Delta$ defective in recombinational repair, indicate that $R A D 14$ shows an epistatic relationship only with $R A D 1$, unlike results obtained with rad14-2, which was probably due to the leakiness of the rad14-2 point mutation. Thus, RAD14 functions in the excision repair pathway.

\section{(4) ScERCC3, the S. cerevisiae homolog of ERCC3 (XP.B)}

An $S$. cerevisiae homologof the human $E R C C 3 / X P B C$ gene has been isolated in Dirk Bootsma's laboratory at Erasmus University in Rotterdam, The Netherlands. It represents a new gene presumably inyolved in excision repair and was cloned by DNA homology using the human $X P B C$ cDNA as a hybridization probe. The Dutch group sent us the yeast gene, which we have designated $S C E R C C 3$, including a partial DNA sequence. We have now completed the DNA sequence of SCERCC.3 and find that the yeast and human-encoded ERCC3 proteins share 52\% identity and $69 \%$ similarity.

Last year, we had constructed a genomic deletion of ScERRC3 and preliminary data suggested that the Scercc3 deletion mutation was a recessive lethal. However, the results were not conclusive since the plasmid for constructing the genomic deletion was generated before the entire nucleotide sequence of the SCERCC3 gene had been completed. We now now that the deletion extended upstream of the translation initiating ATG codon, and the possibility remained that the observed effect was due to another gene tightly linked to ScERCC 3. Since that time, we have constructed a new plasmid for generating a genomic Scercc3 deletion which removes 2116 nucleotides entirely within the SCERCC3 2328 nucleotide long open reading frame. Genetic analysis using this new deletion mutation confirms our previous observation and it is now clear that $S C E R C C 3$ is essential for viability in yeast. Since SCERCC 3 is homologous to human $X P B$, it is very likely that the human XPBC gene is essential for viability as well. The only living representative of XP complementation group B at the time the study by Weeda et al. was published (Cell, 1990, 62:777) contained a frameshift mutation in the last intron-exon junction which resulted in an $X P B$-encoded protein of 781 rather than 782 amino acids, and with the Cterminal 40 amino acids being altered relative to the wild type ERCC.3 protein. We constructed a similar mutation in the SCERCC3 gene in which we introduced a StuI site between residues 797 and 798, resulting in premature termination at residue 798. Thus, this Scercc3(StuI) mutant allele results in a truncated ScERCC3 protein lacking the C-terminal 45 amino acids.. We replaced the wild type $S C E R C C 3$ gene with the mutant $S \operatorname{cercc} 3(S t u \mathrm{I})$ mutation and found that the cells are viable but are UV sensitive. However, the UV sensitivity is not as great as niight be expected, based on the extreme defect in excision repair of the human XPB mutant cells. Thus, as described below, we will isolate additional mutations of $S C E R C C 3$ which confer sensitivity to UV light.

\section{(5) Complex formation between RAD6 and RAD18}

We have further characterized the complex formed in vivo between RAD6 and RAD18 proteins of yeast. The association of RAD6 with RAD18 was detected by co-immunoprecipization. We have found that either of the the human homologs of RAD6, namely HHR6A or HHR6B, can substitute for RAD6 in the immune complex with RAD18. However, the amount of RAD18 found associated with HHR6A or HHR6B is less than with RAD6. These observations provide further evidence for the high degree of conservation in structure and function of RAD6 and the proteins with which it interacts. 


\section{Description of plans for the coming vear:}

\section{RAD3 protein and î́s homologs}

(a) Characterization of rad3 is mutations

Four temperature sensitive ( $t:$ ) for growth mutations of the $R A D 3$ gene of $S$. cerevisiae have been isolated in our laboratory by in vitro mutagenesis with hydroxylamine (HA) of a plasmid containing the RAD 3 gene followed by the "plasmid shuffle" technique. The wild type genomic RAD3 gene has been replaced by each of these $t s$ mutations so that we now have a set of isogenic haploid strains that differ only in the rad 3 allele they carry. The 4 ts mutants will be characterized for their effect on DNA, RNA, and protein synthesis at both the permissive and restrictive temperature. DNA synthesis will also be measured by flow cytometry. Their cell division cycle $(c d c)$ phenotype will be determined by microscopic examination of cells at various times after transfer to the restrictive temperature. Results obtained from these experiments should reveal the cause the conditional inviability of rad3 $t s$ mutants.

$(X P D)$

(b) Complementation of rad3 $4, \operatorname{rad} 3-2$, and other alleles by human ERCC2

Using a cDNA clone obtained from Larry Thompson and Christine Weber at Lawrence Livermore National Laboratory', we have succeeded in expressing the human $E R C C 2$ gene in yeast. The human ERCC2 protein is being purified from these yeast cells and using this construction, we 'will determine whether the ERCC2 gene can complement the UV sensitivity and inviability defects of the radi3-2 and $\mathrm{rad} 3 \Delta$ mutations, respectiveiy. The reciprocal experiment, of determining whether the yeast RAD3 gene can complement the UV sensitivity of XPD cells is being carried out in collaboration with Drs. Thompson and Weber. We will also use several other representative rad3 alleles in these complementation experiments. Since RAD3 and ERCC2 proteins share such extensive amino acid sequence homology, we may find that the two proteins can substitute for one another.

\section{(2) Isolation and characterization of ercc3 ts and $U V^{s}$ mutations}

The SCERCC3 gene, like the RAD3 gene, is essential for cell viability. SCERCC3 encoded protein also contains conserved sequences found in proteins which bind and hydrolyze ATP and are DNA helicases as well. Using the same techniques as mentioned above, we will isolate $t s$ mutations of the SCERCC3 gene by in vitro mutagenesis of the gene with HA followed by plasmid shuffle. The mutants will be characterized for their effect on macromolecular synthesis at the permissive and restrictive temperatures, as well as for their sensitivity to UV. It is hoped that this approach will generate mutants which show mure extreme UV sensitivity than the "XPB-like" mutant described in section 4 above. The most UV sensitive Scercc 3 mutant obtained will be used for determining whether SCERCC3 is required for the incision step of excision repair, or for a subsequent step of excision repair. The UV sensitive mutant will also be used for determination of epistatic interactions with the other members of the RAD3 group, and for synergistic interactions with the RAD6 and RAD52 group mutants.

\section{(3) Isolation of radl mutations}

Both the RAD1 and RADI0 genes, in addition to their role in excision repair of UV damaged DNA, function in a mitotic recombination pathway distinct from the RAD52 recombination pathway. We have obtained evidence for complex formation between RAD1 and RAD10 proteins of yeast by co-immunoprecipitation. During the coming year, the interaction will be studied further by using radl $\Delta$ and radlo $\Delta$ strains as controls. Using in vitro mutagenesis of the $R A D 1$ gene with $\mathrm{HA}$, we will screen for mutants which are interaction deficient, and these 
mutations will be mapped by DNA sequencing. The interaction negative mutants will be characterized for their effect on UV sensitivity and mitotic recombination. This approach is being used with the $R A D I$ gene rather than the $R A D 10$ gene because the probability of success with $R A D 1$ is greater than with $R A D 10$, due to the larger target size of $R A D 1$ (3303 nucleotide $R A D 1$ open reading frame $v s .630$ nucleotide $R A D 10$ open reading frame).

(5) Complementation between $S$, cerevisiae rad14 by the human $X P A C$ gene and of XP complementation group $A$ cells by $R A D 14$

We have obtained the hurnan cDNA encoding the XPAC gene from Dr. K. Tanaka and will determine whether it complements the UV sensitivity of the rad14 $\triangle$ mutant. The XPAC gene will be fused to the highly expressed yeast $A D C l$ promoler, since in our experience, . ADC1::gene fusions express well in yeast. The reciprocal experiment, of determining whether the yeast RAD 14 gene complements the UV sensitivity of complementation group A cells is being carried out by Dr. 'Tanaka, to whom we have sent the RAD14 gene.

\section{(6) Cloning of a huinan homolog of RAD18}

Since we have found that RAD6 and RAD18 proteins form a specific complex in vivo, and that the human HHR6A and HHR6B homologs of RAD6 can substituie for yeast RAD6 and interact with RAD18, it seems likely that there will be a human homolog of yeast RA.D18 which will form a specific complex with human HHR6A and/or HHR6B. We propose to clone the human homolog of RAD18 by using the two-hybrid system developed by Stan Fields and his colleagues (Chien et al., 1991, Proc. Natl. Acad. Sci. 88:9578) to identify and clone genes which encode proteins that interact with a known protein of interest.

\section{PUBLICATIONS (since the last Progress Report was submitted in April 1991)}

Koken, M. H. M., P. Reynolds, I. Jaspers-Dekker, L. Prakash, S. Prakash, D. Bootsma, and J. H. J. Hoeitmakers (1991) Structural and functional conservation of two human homologs of the yeast DNA repair gene RAD6. Proc. Natl. Acad. Sci. USA 88:8865-8869.

Bailly, V., P. Sung, L. Prakash, and S. Prakash (1991) DNA.RNA helicase activity of RAD3 protein of Saccharomyces cerevisiae. Proc. Natl. Acad. Sci. USA 88:9712-9716.

Prakash, S. and L. Prakash (1992) Conservation of structure and function of DNA repair genes between yeast and human. Rad. Res. vol, II. Congress Proceedings, pp. 239-244, (ed. by W. C. Dewey, M. Edington, R. J. M. Fry, E. J. Hall, and G. F. Whitmore), Academic Press, San Diego, CA.

Bankmann, M., L. Prakash, and S. Prakash (1992) Yeast RAD14 and human xeroderma pigmentosum group A DNA-repair genes encode homologous proteins. Nature 355:555558.

Sung, P., L. Prakash, and S. Prakash (1992) Renaturation of DNA catalysed by yeast DNA repair and recombination protein RAD10. Nature 355:743-745.

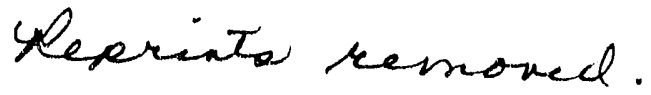




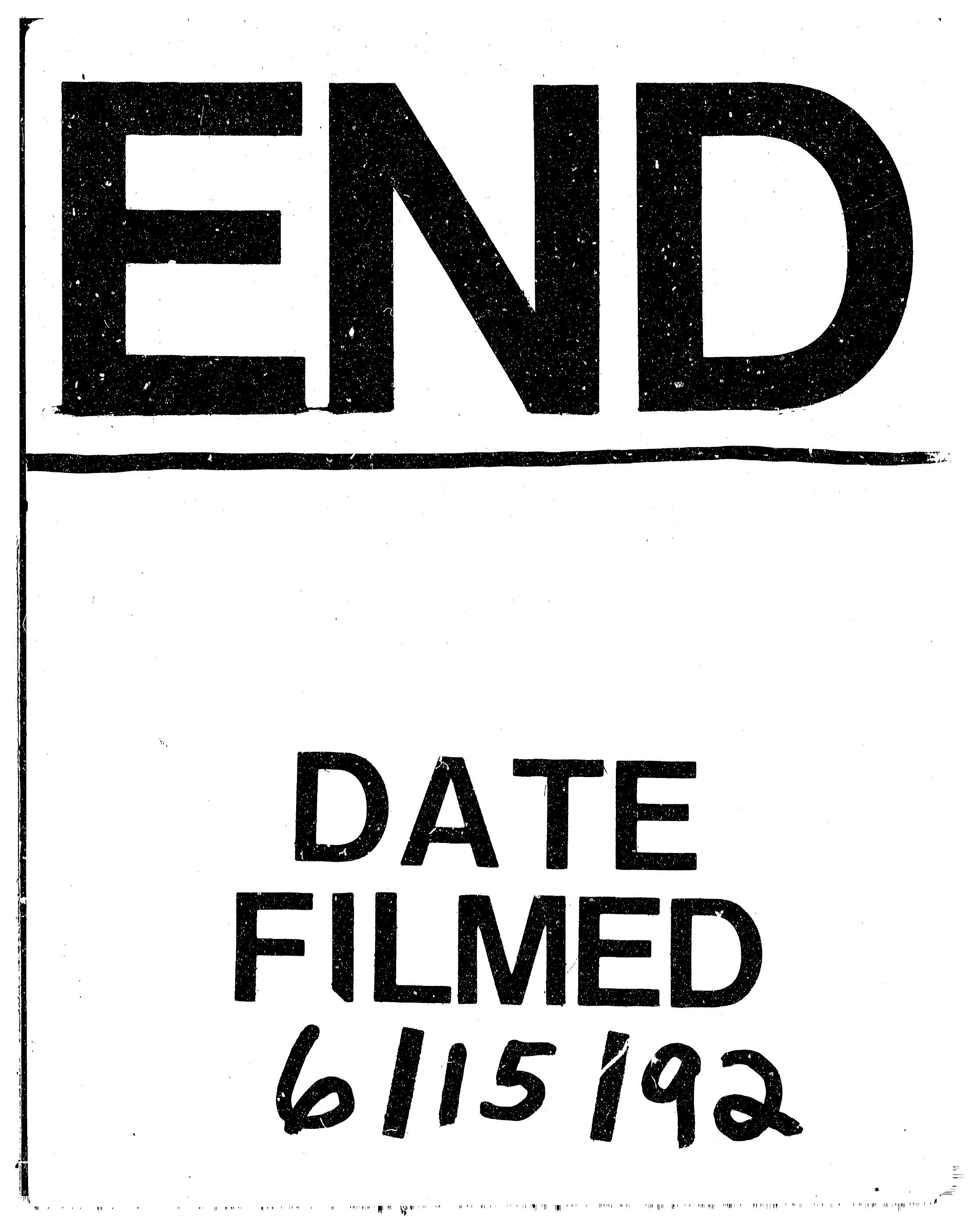




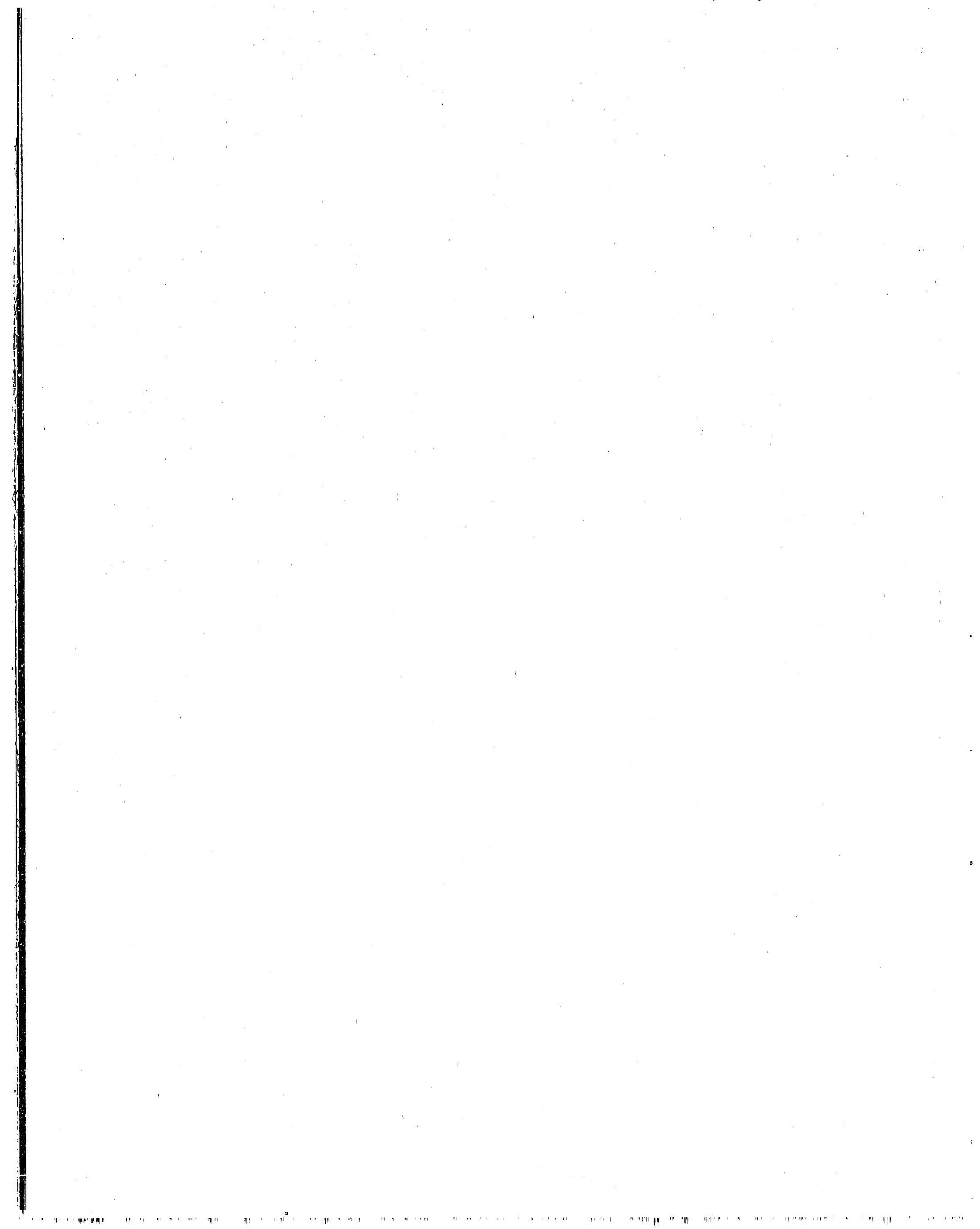

